\title{
Perspectivas sobre la educación financiera, su importancia e impactos de su incorporación en los niveles educativos
}

Nitzia Vázquez Carrillo

Universidad Nacional Autónoma de México

México

Manuel Díaz Mondragón

Universidad de La Salle

México 


\title{
Perspectivas sobre la educación financiera, su importancia e impactos de su incorporación en los niveles educativos
}

\author{
Nitzia Vázquez Carrillo ${ }^{1(D)}$ \& Manuel Díaz Mondragón ${ }^{2}$ (i) \\ 1 Universidad Nacional Autónoma de México - México \\ 2 Universidad de La Salle - México
}

Para citaciones: Vázquez Carrillo, N., \& Díaz Mondragón, M. (2021). Perspectivas sobre la educación financiera, su importancia e impactos de su incorporación en los niveles educativos. Panorama Económico, 29(2), 102116.

Recibido: 12 de noviembre de 2020

Aprobado: 16 de febrero de 2021

Autor de correspondencia:

Nitzia Vázquez Carrillo

nitziavaca@economia.unam.mx

Editor: Andrés Escobar E. Universidad de Cartagena-Colombia.

Tipología IBN Publindex: Artículo de Revisión

Copyright: (C) 2021. Vázquez Carrillo, N., \& Díaz Mondragón, M. Este es un artículo de acceso abierto, distribuido bajo los términos de la licencia https://creativecommons.org/licenses/bync-sa/4.0/ la cual permite el uso sin restricciones, distribución y reproducción en cualquier medio, siempre y cuando que el original, el autor y la fuente sean acreditados.

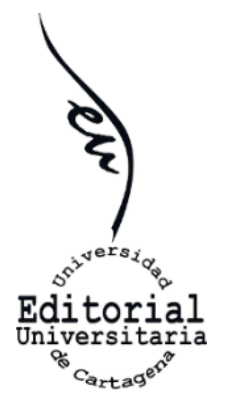

\section{Perspectives on financial education, its importance, and impacts of its incorporation in the educational levels}

\begin{abstract}
This article is the result of research related to financial inclusion and the financial education training process. Its objective is to present an argumentative analysis on the importance and impacts of incorporating financial education from basic to professional education. It also seeks to make contributions from the historical perspective on the development of the subject at an international level and from the conceptual level, providing definitions of education, culture and financial inclusion that serve as a basis for future explorations and analysis. An argumentative analysis of the benefits that are unleashed from the personal and family, corporate level and finally at the country level with transversal repercussions. Benefits are discussed with a focus on issues related to the use of money, the administration of income and expenses, the alternatives of savings, investment and means of financing, financial management in the long term, elements inherent to financial decisions and reduction of vulnerabilities. In this sense, the transmission of knowledge on financial education from an early age provides long-term benefits, triggering improvements in decision-making both at the micro and macro levels. It allows an improvement in the management of financial services,
\end{abstract}


make informed saving decisions about savings, investment, financing, and provides instruments for the protection of users, which as a whole reduces the vulnerabilities of economic actors.

Keywords: Financial education; financial inclusion; financial development; financial policies; financial markets.

\section{INTRODUCCIÓN}

El artículo tiene como propósito presentar un análisis argumentativo sobre los aportes de la educación financiera, relevancia e impactos de incorporarla en los programas de estudio de todos los niveles educativos, desde una investigación ${ }^{1}$ de tipo teóricodescriptiva con el fin de contribuir a la profundización del conocimiento técnico, así como el diseño y aplicación de políticas públicas en materia de educación e inclusión financiera. En la siguiente sección se presenta la metodología utilizada; posteriormente se aborda la relevancia de la educación tradicional en conjunto con la formalización de estudios en materia financiera; incluyendo como valor agregado y resultado investigativo la propuesta de conceptos relevantes tales como alfabetización financiera; educación financiera y también, cultura financiera. La tercera sección presenta una síntesis histórica del desarrollo de la educación e inclusión financiera a nivel internacional y su promoción por parte de organismos financieros internacionales. La cuarta sección presenta el análisis argumentativo de los beneficios de la educación financiera considerando efectos transversales y que robustece nuestro aporte a la materia seccionando sus posibles impactos en siete temas que son: uso del dinero; administración de ingresos y gastos; alternativas de ahorro, inversión y medios de financiamiento; gestión financiera en el largo plazo; elementos inherentes a las decisiones financieras y disminución de vulnerabilidades. Finalmente, se presentan las principales conclusiones que señalan que la transmisión de conocimiento en materia de educación financiera desde edades tempranas brinda beneficios en el largo plazo para todos los usuarios de los servicios financieros desencadenando mejoras a en la toma de decisiones tanto a nivel micro y como macroeconómicos, porque permite mejorar la gestión del medio de intercambio, asumir decisiones informadas en materia de ahorro, inversión, financiamiento, provee de instrumentales para la protección de los usuarios, lo que en su conjunto reduce las vulnerabilidades de los actores económicos.

\section{MATERIALES Y MÉTODOS}

La investigación es de tipo teórico-descriptiva cuyo propósito es contribuir al bagaje financiero y a extender el conocimiento en materia de educación e inclusión financieras. Para tal fin, se fundamenta teóricamente el proceso educativo y su importancia; se reseña históricamente el surgimiento y avance de los programas y políticas gubernamentales impulsados a nivel internacional en la materia; y se aportan al argot del tópico con propuestas conceptuales específicas para cultura financiera, alfabetización financiera y educación financiera.

1El presente artículo forma parte de los avances en los proyectos de investigación "Inclusión Financiera en México" (PAPIIT No. IA302221) y "Elaboración de materia didáctico para el estudio de indicadores económicos y financieros" (PAPIME No. 303021), financiados por la Universidad Nacional Autónoma de México. 
También se brindan argumentos que describen y evidencian los beneficios e impactos de incorporar la educación financiera desde la primera infancia y en los programas de estudio de educación básica y su continuación en todas las áreas de conocimiento de los planes de estudio de educación superior. El marco de referencia considera elementos teóricos e históricos incluidos como parte de los apartados relativos a educación financiera vs formación tradicional y al desenvolvimiento cronológico de la temática.

\section{DE LA FORMACIÓN EDUCATIVA TRADICIONAL A LA EDUCACIÓN FINANCIERA}

En cualquier momento aprender y educarnos resulta de vital importancia porque ello brinda diversidad de ventajas, no solo se trata de acumular información sino de un proceso complejo por el cual el conocimiento se va enriqueciendo al tiempo que modifica y cuestiona lo aprendido previamente, además de generar nuevas competencias. En este sentido, ya no se cuestiona la relevancia de formación desde la educación básica hasta lograr el dominio de algún oficio o disciplina profesional. Sobre el significado e importancia de la educación es posible encontrar infinidad de aportaciones como la de Valenzuela (2010) quien menciona:

La educación es un proceso gradual mediante el cual se transmiten un conjunto de conocimientos, valores, costumbres, comportamientos, actitudes y formas de actuar que el ser humano debe adquirir y emplear a lo largo de toda su vida, además diversas formas de ver el mundo que nos rodea; todo ello para poder desenvolvernos de manera activa y participativa en sociedad (p. 2).

Por otra parte, relacionando la educación con el bienestar nacional y personal, Narro, Martuscelli y Barzana (2012) señalan:

La educación es uno de los factores que más influye en el avance y progreso de personas y sociedades. Además de proveer conocimientos, la educación enriquece la cultura, el espíritu, los valores y todo aquello que nos caracteriza como seres humanos. La educación es necesaria en todos los sentidos. Para alcanzar mejores niveles de bienestar social y de crecimiento económico; para nivelar las desigualdades económicas y sociales; para propiciar la movilidad social de las personas; para acceder a mejores niveles de empleo; para elevar las condiciones culturales de la población; para ampliar las oportunidades de los jóvenes; para vigorizar los valores cívicos y laicos que fortalecen las relaciones de las sociedades; para el avance democrático y el fortalecimiento del Estado de derecho; para el impulso de la ciencia, la tecnología y la innovación. (p.35)

Para el último cuarto del siglo XX dentro de las disciplinas de estudio económico y financiero surgió la propuesta relativa a las microfinanzas, antecedente directo de la educación e inclusión financieras. Las microfinanzas establecieron un fundamento teórico y una práctica a nivel internacional comprendiéndolas como conjunto de organismos cooperativos cuya tarea era otorgar senvicios financieros a la población pobre y aquella que ha sido excluida del sistema financiero. Como encomienda secundaria debían crear instrumentos de ahorro, financiamiento y seguros con características especiales los analfabetos, los que no poseen garantías, sus montos de ahorro son mínimos e inconstantes, los períodos de pago y capacidad de retorno del capital pueden ser extensos e irregulares. 
Posteriormente estos temas en la ciencia económica se popularizaron, aunque denominándolos de muy distintas formas desde desarrollo financiero, profundización, amplitud y masificación financieras, bancarización, finanzas para personas en la base de la pirámide por ingresos, finanzas populares, banca social, banca del pueblo (Armendáriz y Morduch, 2006; Robinson, 2001).

Con mayor énfasis desde principios de la década de los ochentas, a nivel internacional se manifestó una preocupación y disposición por fomentar el aprendizaje en materia financiera sin importar el nivel educativo, la profesión o actividad realizada, bajo el supuesto de que los conocimientos financieros son necesarios para toda persona porque diariamente toman decisiones financieras que tienen impactos en la gestión de su patrimonio. Éstos esfuerzos se convirtieron en campañas de difusión, programas, conferencias, seminarios y otros eventos de difusión masiva, instituciones especializadas o al menos enfocadas a la temática y por supuesto, inclusión de materias financieras en programas escolares de niveles educativos y especialidad diversa. Por supuesto en contextos de integralidad y proveer de marcos de referencia más robustos surgieron las derivaciones desde la educación hacia los procesos de enseñanza y aprendizaje, en este sentido retomamos los aportes de la Secretaría de Educación Pública (2017a) como autoridad y organismos supervisor de la política educativa en México, que los define así:

Enseñanza. Proceso didáctico orientado al desarrollo de conocimientos o habilidades por la vía de acciones -intelectuales, sociales o físicas- que faciliten al estudiante la aprehensión del contenido. Aprendizaje proceso cognitivo individual, la enseñanza ofrece el contenido de los modos más convincentes para que la acción propia de los estudiantes los incorpore. (p. 201).

Al mismo tiempo surgieron términos y bagaje que complementaria la ya abundante jerga financiera; de manera específica se empezaron a emplear términos brindándoles un significado diferente según el país, la institución o el sector que lo comenzaba a utilizar. Es así como se empezó a hablar, por ejemplo, de alfabetización, cultura y educación financieras, cuyas definiciones presentamos a continuación considerando como fuentes a instituciones internacionales ampliamente involucradas en su diseminación o bien, literatura técnica cuyos aportes hemos considerado amplios y profundos al abordar el tema. Para la Organización para la Cooperación y el Desarrollo Económico, la alfabetización financiera es una combinación de conciencia, conocimiento, habilidad, actitud y comportamiento necesarios para tomar decisiones financieras acertadas $y$, en última instancia lograr el bienestar financiero individual (OCDE, 2012). En tanto que la organización australiana encargada del desarrollo de habilidades financieras afirma que la alfabetización financiera consiste en capacitar a las personas para que tomen decisiones informadas y seguras sobre todos los aspectos de su presupuesto, gasto y ahorro, así como sobre el uso de productos y servicios financieros, desde las operaciones bancarias cotidianas hasta los préstamos, las inversiones y la planificación para el futuro. (FC, 2003).

Autores como Cruz (2018) afirman que la alfabetización financiera implica un proceso que proporciona herramientas necesarias para comprender e interpretar el mundo económico. No obstante, utiliza el término como sinónimo de educación financiera sin acotar o enfatizar diferencias. Para el término de educación financiera el mismo organismo de cooperación internacional (OCDE) señala que puede definirse como el 
proceso mediante el cual los consumidores/inversores financieros mejoran su comprensión de los productos, conceptos y riesgos financieros y, a través de la información, la instrucción y/o el asesoramiento objetivo, desarrollan las habilidades y la confianza para ser más conscientes de los riesgos y las oportunidades financieras, para tomar decisiones con conocimiento de causa, para saber dónde acudir en busca de ayuda y para tomar otras medidas eficaces para mejorar su bienestar financiero (OCDE, 2005; 4). Hernández y Soraya (2019) la conceptualizan como el proceso mediante el cual las personas van adquiriendo formación e información, para desarrollar conocimientos propios que les permitan analizar las oportunidades y tomar decisiones financieras asertivas, de manera que puedan mejorar su calidad de vida y el bienestar de su entorno. Además, elaboran una propuesta que afirma que para mejorar las decisiones financieras de los ciudadanos se requiere incorporar la Educación Financiera como un derecho ciudadano y tanto el Estado, como las Empresas y la Sociedad Civil deben asumir su cuota de responsabilidad en la construcción de inteligencia financiera colectiva.

Otros autores la definen con base en su uso aplicación, Ortega et al (2009) señalan que se trata de potenciar conocimientos, destrezas y habilidades de corte económico que permitan al alumnado comprender la importancia de conceptos clave como el ahorro, el presupuesto, los gastos, los ingresos, el coste de las cosas, la calidad de vida, el consumo responsable, etc., así como poder llevar a cabo procedimientos bancarios básicos como la apertura de una cuenta, el control de sus propios ingresos, cambios de divisas, uso de tarjetas de crédito y de débito. En último término, el objetivo es que sean capaces de extrapolar dichos conocimientos y habilidades a su vida cotidiana, personal y familiar. Paulatinamente desde inicios del siglo XXI se configuraron políticas públicas entorno de los tópicos educación e inclusión financiera. En el caso colombiano se ha instaurado a nivel nacional a través del Pan Nacional de Desarrollo se señala que incluye la educación económica y financiera es el proceso a través del cual los individuos desarrollan los valores, los conocimientos, las competencias y los comportamientos necesarios para la toma de decisiones financieras responsables, que requieren la aplicación de conceptos financieros básicos y el entendimiento de los efectos que los cambios en los principales indicadores macroeconómicos generan en su propio nivel de bienestar económico (PND, 2010).

Existen coincidencias con lo relativo a cultura financiera, dado que se utiliza indistintamente dicho término con el de educación financiera, como si fuesen sinónimos y cuyo significado no difirieran, lo cual puede considerarse un error. Por lo tanto, es necesario establecer sus diferencias conceptuales: Cultura financiera es el conjunto de conocimientos y manifestaciones de las personas en las que basan sus decisiones financieras, considerando un entorno financiero informal y usando como evidencia y sustento de tales decisiones aspectos subjetivos como preferencias personales, consejos de conocidos, familiares o amigos, así como, la experiencia propia ordinariamente desligada del conocimiento técnico. Por otro lado, educación financiera es el proceso de preparación formal, absorción de conocimientos financieros que le permitan a los seres humanos desarrollar habilidades, aptitudes y competencias para la lectura, interpretación y evaluación de datos e información financiera; como resultado final le capacita para tomar decisiones financieras informadas y acordes a su realidad, objetivos financieros y perfil propios, además de que le posibilitan el acercamiento a los sistemas financieros y el acceso y uso de instrumentos, productos y servicios ofrecidos en éstos para el ahorro, 
la inversión, el financiamiento, el retiro, los seguros, entre otros. Forma parte de las políticas gubernamentales cuyo objetivo es acrecentar el nivel de conocimiento además del acceso y uso del sistema financiero entre la población.

La inclusión financiera se entiende como el conjunto de políticas públicas que deben tener como fines: conformar sistemas financieros amplios y competitivos, fomentar el acceso y uso de servicios y productos financieros universales porque éstos fungen como lubricantes a los engranajes productivos y comerciales; brindan poder económico y social a los agentes económicos, lo que a su vez permea el crecimiento económico y la distribución del ingreso al crear canales de aporte de recursos para el sector productivo. De esta manera nuestra definición no solo hace referencia a la infraestructura sino también promover su uso y conocer las causas de que la gente se aparte del sistema financiero, lo cual no siempre se debe a falta de infraestructura. También, vislumbrar la inclusión financiera como mecanismo de empoderamiento, que debe ser abordado como un plan gubernamental diseñado y aplicado como política pública y finalmente, relacionarlo con el crecimiento y el ingreso.

\section{ESBOZO HISTÓRICO DE LA EDUCACIÓN FINANCIERA}

Resulta una tarea compleja el datar con precisión cuándo y quiénes comenzaron los esfuerzos en torno a la educación financiera porque es un proceso continuo, aunque asincrónico entre naciones, ensalzado en ocasiones por el sector público y en otras por el privado o una combinación de ambos. En este sentido, la Coalición Jumpstart se fundó en 1995, a partir de una idea atribuida a William E. Odom, Presidente y Director General de la Ford Motor Credit Corporation. En su primera reunión, organizada por H. Randy Lively, Presidente y Consejero Delegado de la Asociación Americana de Servicios Financieros, establecieron como objetivo "desarrollar un plan estratégico para mejorar la calidad y el alcance de los módulos curriculares para la educación en finanzas personales en las escuelas de Estados Unidos desde el jardín de infancia hasta el $12^{\circ}$ grado" (Jumpstart, 2020, p. 45)

La organización Financial Capability (CF) es gestionada por el Departamento del Tesoro de Australia, y es la entidad encargada de la política de educación financiera en Australia. Elabora y conduce en conjunto con la empresa Roy Morgan Research encuestas en materia de educación financiera desde 2003 (FC, 2003). Desde el primer estudio se ha enfocado en tres áreas financieras:

\begin{tabular}{|c|c|c|}
\hline $\begin{array}{l}\text { Comprensión Financiera: } \\
\text { Comprensión de lo que es } \\
\text { el dinero y cómo se } \\
\text { intercambia. Comprender } \\
\text { de dónde viene y a dónde } \\
\text { va el dinero }\end{array}$ & $\begin{array}{l}\text { Competencias Financieras: } \\
\text { Principales características de los } \\
\text { servicios financieros básicos. } \\
\text { Registros financieros y apreciar la } \\
\text { importancia de leerlos y } \\
\text { comprenderlos. } \\
\text { Actitudes ante el gasto de dinero y } \\
\text { el ahorro. } \\
\text { Conocimiento de los riesgos } \\
\text { asociados a algunos productos } \\
\text { financieros y apreciación de la } \\
\text { relación entre el riesgo y la } \\
\text { rentabilidad. }\end{array}$ & 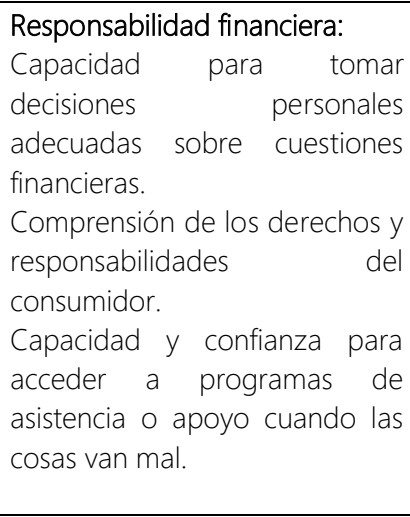 \\
\hline
\end{tabular}


Desde comienzos del nuevo milenio se fueron multiplicando los esfuerzos institucionales, programas, y políticas gubernamentales a nivel nacional e internacional en pro de la educación financiera, en la mayoría de las ocasiones, en combinación con la inclusión financiera. Abordarlas en su totalidad no forma parte de los objetivos al presentar nuestra investigación y resultados preliminares, no obstante, abordamos los que hemos considerado más relevantes con base en su extensión, logros y actualidad. La OCDE en 2005 emitió un documento intitulado Recommendation on Principales and Good Practices for Financial Education en el cual definió por primera ocasión la educación financiera y tres años después, en 2008 creó la Red Internacional de Educación Financiera (INEF, siglas en inglés correspondientes a: International Network on Financial Education) existente aún en el 2021, cuyo objetivo es apoyar a autoridades de diversos países para diseñar y aplicar políticas públicas en materia de educación financiera. En el 2006 surgió un programa denominado Programa Global de Educación Financiera elaborado por la organización sin fines de lucro Microfinance Opportunities and Freedom from Hunge, que a su vez es patrocinado fundamentalmente por la fundación Citi del grupo financiero internacional del mismo nombre. Resultó novedoso tanto por su enfoque en las personas que por nivel de ingresos se hayan en el umbral de pobreza extrema como por generar programas y planes de estudio específicos en materia de educación financiera. Al 2021 sigue en operación conformando redes de trabajo con otras instituciones financieras de modo que éstas ofrezcan cursos e información gratuita al mismo tiempo que ofertan sus servicios financieros.

La Comisión Europea también cuenta con iniciativas y programas en materia de educación financiera, de modo específico en el 2007 dio a conocer ocho principios básicos para planes de educación financiera de alta calidad (CEE, 2007); luego en 2008 se creó el Grupo de Expertos en Educación Financiera (EGFE por sus siglas en inglés) para la operación de programas y difusión de la educación financiera en la Comunidad Europea. Igualmente solicitó la realización de un estudio sobre las condiciones y nivel de conocimiento financiero en los estados miembros, cuyos resultados se dieron a conocer el mismo año (Habschick, Seidl, Evers, 2007) y desarrolló a través de la Dirección General de Sanidad y Consumo el programa DOLCETA, el acrónimo para Development of On Line Consumer Education Tools for Adults cuyo traducción sería Desarrollo de herramientas de educación del consumidor en línea para adultos, que mediante su página web difunde los derechos del consumidor y los servicios financieros entre otros programas.

Como consecuencia de tales esfuerzos por la Comunidad Económico Europea, a nivel nacional cada miembro de la comunidad ha impulsado diversidad de tareas en la materia, algunas de ellas las ejemplificamos a continuación:

> Alemania fundó un promotor denominado Sparkassen-Finanzgruppe - se traduciría como Agrupación de Cajas de Ahorro -, que conjunta a cajas, bancos y otras instituciones financieras y entre sus objetivos estratégicos se halla la educación financiera.

> Irlanda llevó a cabo la constitución de un organismo público denominado Agencia Nacional del Consumo (NCA, por sus siglas en inglés), su propósito general es salvaguardar los derechos de los consumidores de servicios 
financieros y entre sus tareas se estipula el desarrollo de programas para dotar de habilidades financieras.

$>$ En Francia la tarea se ha llevado a cabo de manera conjunta entre el Ministerio de Economía, Industria y Empleo y el Instituto de Educación Financiera Pública (IEFP, por sus siglas en Francés); de este último destacan dos acciones que han tenido amplia difusión y aceptación entre las personas: en 2009 publicó el texto Finanzas personales para ineptos" y a través de su sitio web emite un programa llamado Finanzas para todos. De manera conjunta, el IEFP y el Ministerio, imparten seminarios, cursos y pláticas gratuitas para difundir la educación financiera.

> Italia, lo relacionado con difundir y gestionar la política de educación financiera se legisló por el senado y encargado al Ministerio de Educación. Además, los bancos italianos se han agrupado en un organismo conocido como Pattichiari Consortium cuyo objetivo es que los consumidores de productos financieros tomen decisiones informadas.

> En Hungría la labor fundamentalmente la lleva a cabo su banco central llamado Magyar Nemzeti Bank, en 2004 inauguró el Centro de Visitantes para la educación financiera, desde 2005 organiza eventos diversos para su difusión, en 2007 incluyo la temática en sus programas de estudios y se llevan a cabo competencias escolares sobre nivel de conocimientos financieros.

> Austria lleva a cabo actividades de difusión a través de su banco central Oesterreichische Nationalbank, se inauguró el Museo del Dinero y ha conformado programas de formación en línea con páginas dedicadas a la educación financiera, difusión de materiales educativos, juegos interactivos, simuladores y una plataforma de conexión con el banco central que promete atender y resolver cualquier duda en la materia.

Canadá en 2009 generó el Grupo de Trabajo de Expertos en Educación Financiera su primera labor fue brindar 30 recomendaciones en materia de educación financiera, fue hasta 2013 cuando se publicó la Ley Financial Literacy Leader que coordinaría los esfuerzos a nivel nacional para promover la alfabetización financiera. (Sorenson, 2021)

En México forma parte de una política pública a nivel nacional formalizada a través de incorporación en la Ley para regular las agrupaciones financieras y con ello creando al Consejo Nacional de Inclusión Financiera (CONAIF) y el Comité de Educación Financiera (CEF), específicamente abordados en capítulos III y IV, artículos 183 al 192. Además, se establece en la Política Nacional de Inclusión Financiera que en 2030 se logrará:

Que las y los mexicanos sin importar su edad, sexo, ingreso, condición laboral ni lugar donde radiquen, accedan y usen los productos y servicios del sistema financiero, con mejores conocimientos, habilidades y aptitudes económicofinancieras. En el sistema financiero se incorporan a los territorios, grupos o empresas históricamente sub-atendidos o excluidos tales como población rural, mujeres, indígenas, personas adultas mayores, migrantes, y micro, pequeñas y medianas empresas (p.48).

En este orden de ideas, la política en la materia se ha traducido en la obligatoriedad de desarrollar contenidos de educación financiera según consta en el Modelo Educativo para la Educación Obligatoria, diseñado y publicado por el ente público a cargo del sector 
educativo nacional. Destacando que en México la Carta Magna en su carácter de ley máxima prescribe en su artículo tercero como niveles educativos obligatorios a la educación inicial, preescolar, primaria, secundaria y media superior. (CPEUM, 1917).

En el 2015 la Organización de las Naciones Unidas en conjunto con líderes de múltiples naciones se pusieron de acuerdo para llevar a cabo acciones que permitiesen en el plazo de 15 años el logro de 17 objetivos de desarrollo sostenible (ODS) cuyo cumplimiento conjunto permitiría un futuro sostenible. En esta temática a la educación financiera se le atribuye un doble papel, primero como factor transversal, es decir, herramienta que incide en el logro del total de los objetivos propuestos y segundo, el énfasis en la consecución del objetivo 4 Educación de calidad; de manera específica este ODS pretende lograr que todos los menores terminen la educación primaria y secundaria; que la educación básica sea gratuita, equitativa y de calidad; acceso igualitario a formación de nivel técnico o superior; todas las personas estén alfabetizados y desarrollen competencias en lectura y matemáticas; generar entornos de aprendizaje seguros, libres de violencia e inclusivos. Todo lo anterior como mecanismo de movilidad social.

De este modo, la educación financiera se convierte en pivote en cada uno de los ODS además del énfasis para el logro de los propósitos en materia educativa, porque el acceso, uso de servicios financieros así como desarrollar habilidades, aptitudes y competencias para la lectura, interpretación y evaluación de datos e información financiera; que como resultado final capacita para tomar decisiones financieras informadas permea el grado de avance y consecución de tales propósitos para el bien y protección de la humanidad.

\section{ANÁLISIS DE LOS BENEFICIOS DE LA EDUCACIÓN FINANCIERA}

Los beneficios se ven reflejados en modo de cascada que desencadena sus efectos paulatinamente desde lo personal hacia lo familiar; lo corporativo y finalmente, a nivel de naciones; porque se trata de una serie de conocimientos cuya aplicación es transversal para el ser humano sin importar su nivel educativo, actividad desempeñada o área de profesionalización, nacionalidad, grupo etario o género. Luego de la revisión y estudio en materia de educación financiera, los aportes a la materia mediante un análisis que por temáticas señala los beneficios de la educación financiera relacionado con: uso del dinero; administración de ingresos y gastos; alternativas de ahorro, inversión y medios de financiamiento; gestión financiera en el largo plazo; elementos inherentes a las decisiones financieras y disminución de vulnerabilidades.

El dinero ha sido ampliamente estudiado, desde la perspectiva histórica pasando por sus formas, proceso de fabricación, funciones, características e impactos económicos; no obstante, en materia de educación financiera nos enfocaremos en dos vertientes; primero, el costo que representa su producción y circulación. Segundo, el impacto de que al ser un instrumento que le brinda derechos a su tenedor, entonces es objeto constante de delitos como la falsificación, malversación y como mecanismo para financiar innumerables actos y actividades ilícitas. Jeftanovic (2001) aduce que los motivos del auge en el uso de las distintas formas de dinero electrónico por el lado del usuario se deben a factores más simples como la comodidad, seguridad, en tanto que, por el lado del 
productor, coincide con nosotros en que reside al bajo costo de operación y en resultar un obstáculo a transferencia de fondos ilegítimos.

En este sentido una respuesta innovadora para reducir tanto su costo como las posibilidades de delinquir, ha sido la implementación de dinero y medios de liquidación electrónica. Lo que significa que el mismo dinero de curso legal en cada economía, emitido por los respectivos bancos centrales se hace circular mediante el sistema de pagos definido como: el conjunto de instrumentos, procedimientos bancarios y, por lo general, sistemas interbancarios de transferencia de fondos que aseguran la circulación del dinero (Banxico, 2020).

A medida que se generaliza el uso de dinero no físico entonces es posible reducir el monto de billetes y monedas en circulación, según los bancos centrales el costo por billete de cualquier denominación oscila entre 0.10 a 0.90 centavos ello expresado en las unidades monetarias que correspondan. Dicha cifra resulta muy pequeña, pero si considera que se deben fabricar miles de millones o más billetes entonces el resultado asciende entre 100 y 900 millones de unidades monetarias tan solo por fabricar, haría falta agregar el costo de distribución, además de que derivado de su circulación se deberán reemplazar las piezas dañadas. Entonces, las naciones pueden reducir estos montos y aplicarlos en otras áreas de gasto al propiciar el uso de dinero no físico.

Asimismo, el circulante electrónico y los sistemas de pagos que permiten su trasvase registran las operaciones realizadas, haciendo más difícil la inclusión en los sistemas financieros de dinero procedente de actividades ilícitas, lo que les aporta seguridad a los usuarios y les reduce riesgos a los sistemas financieros.

Sobre administración de ingresos y gastos cuando las personas son capaces de elaborar un presupuesto que incluya el monto total y las fuentes de sus ingresos, así como sus gastos igualmente su cuantía y porque conceptos, se percatan de como realizan la distribución de los mismos, que rubros pueden ser aumentados y disminuidos, lo que en conjunto representa una planificación en el corto plazo. De ello deriva también la posibilidad de adquirir hábitos de ahorro, consumo reflexivo evitando realizar compras que únicamente atienden al gusto, moda, capricho, pánico u otras conductas irracionales, en cambio, permite decisiones basadas en el análisis para mejorar la distribución tanto de la renta percibida como de los desembolsos llevados a cabo. Esta herramienta presupuestal puede combinarse con otros conocimientos técnicos como la inflación o el costo del dinero, lo que les permite a sus usuarios llevar la planeación del corto al mediano y largo plazos, la relevancia y utilidad de elaborar presupuestos queda de manifiesto en la multiplicidad de programas, cursos y materiales de educación financiera que lo incluyen como parte de las herramientas básicas a enseñar, (evidencia de ello se pueden consultar SERNAC, S/A; WWB, 2008; Condusef, 2009).

En materia de alternativas de ahorro, inversión y medios de financiamiento conlleva el conocer, familiarizar a los usuarios con el entorno financiero disponible, esto es, las elecciones financieras posibles para el ahorro, la inversión, el financiamiento y las instituciones que las ofrecen. Lo anterior en realidad forma parte de todo un proceso que inicia en la parte educativa formal, la generación de hábitos, continua con el acercamiento a los sistemas financieros por parte de los usuarios y finaliza con el uso de los 
instrumentos y productos ofertados. Requiere el conocimiento necesario para distinguir entre ahorro, inversión y financiamiento, así como allegarse de información que permita evaluar las opciones y seleccionar el más viable acorde a las condiciones personales, familiares y corporativas que se presenten. En suma, todo redunda en que el conocimiento y su aplicación en la toma de decisiones a nivel micro o personal propiciará mayores niveles de ahorro e inversión en una economía al tiempo que para familias y empresas representa el logro de objetivos y metas financieras; además aportará más opciones de financiamiento lo que impactará el nivel de competencia y concentración de los sistemas financieros al intentar brindar novedades al sector; lo que para quienes requieren de recursos les permitirá elegir mejor, entre más productos y considerando elementos técnicos como tasa de interés, plazo y sus propias posibilidades de pago. Igualmente, en la medida que existan fondos disponibles que sean otorgados y aplicados en inversiones productivas entonces ello se verá reflejado en el aumento de proyectos, emprendimientos, por consecuencia más empleo, consumo y un círculo virtuoso que en largo plazo incentive mayores niveles de crecimiento económico.

La gestión financiera para el largo plazo implica poseer habilidades y competencias suficientes para establecer objetivos y metas financieras claras, específicas unido a conocimiento técnico financiero aplicado en por ejemplo fondos para el retiro o el uso de seguros y por supuesto haber desarrollo hábitos y disciplina que facilite el cumplimiento de los propósitos impuestos. Al respecto Cruz (2018) evidencia que acostumbrar a los menores de 12 años a planear financieramente y formarse hábitos para el ahorro tiene una relación directa con el contexto y costumbres familiares; concluye que se trata de aprendizajes intergeneracionales. Por otra parte, se debe considerar que en cualquier actividad que desempeñemos llegará el momento, sea por accidente o paso del tiempo, que no podremos llevarla a cabo más. El nivel de ingresos a lo largo de nuestras vidas fluctuará por cambios en fuente de empleo, posición en el mismo o alguna circunstancia que afecte nuestra salud, entre otras razones. En cualquier caso, es necesario planear para el futuro, procurando poseer recursos suficientes para el sostenimiento en la vejez o enfrentar adversidades. Para ello, los sistemas financieros suelen ofrecer opciones de inversión de los fondos para el retiro los cuales pueden ser públicos o privados, así como diversidad de seguros como protección ante la materialización de un riesgo como la muerte, enfermedades, accidentes, entre otros. Tratándose de fondos para el retiro resulta fundamental planear el monto total requerido en función de la pensión final deseada, ello brindará datos sobre el nivel de ahorro requerido durante la vida laboral activa. Igualmente es necesario estar al tanto de costos y gastos financieros por operación de dichos fondos, la fecha y requisitos a cumplir para que estén disponibles, quienes pueden ser los beneficios de dichos recursos y los rendimientos aportados por ese ahorro que normalmente en la actualidad ha quedado a cargo de inversionistas institucionales. Relativo a los seguros será necesario contemplar los riesgos que cubren, su monto, la prima o pago requerido a cambio de dicha protección, condicionantes y beneficios adicionales, requisitos para su cobro en caso del suceso protegido.

Los elementos inherentes a las decisiones financieras, elegir entre un curso de acción dado requiere poseer información; realizar un proceso de evaluación de la misma; intentar, siempre que sea posible, reducir sesgos, subjetividades y actuar por impulso o atendiendo únicamente a los sentimientos. Siempre que las personas tengamos presente 
estas directrices entonces demandaremos a las autoridades, instituciones y en general, a los sistemas financieros el transparentar la información y proveernos de mayor cantidad de datos comprensibles que nos permitan hacer mejores evaluaciones y comparativos. En conjunto, los miembros de las sociedades pedirán más y mejor información, al mismo tiempo surgirán mejores herramientas e instrumentales que apoyen el análisis de dicha información como simuladores, aplicaciones, folletos informativos, indicadores de desempeño. Este rubro en realidad impacta el resto de los beneficios descritos debido al conocimiento que brinda lo que a su vez formará usuarios de los sistemas financieros más analíticos, exigentes y perspicaces los instrumentos y productos que les ofrecen. Sobre este punto Bosch et al. (2018) con el fin de evaluar el impacto de recibir educación financiera como previsión para el largo plazo encuentran que sus conocimientos financieros se fortalecen ampliamente, aunque no les es posible brindar resultados concluyentes en cuanto a la traducción de ello hacia mejoras en el estado financiero personal.

Finalmente, aunque no menos relevante es la disminución de vulnerabilidades conocer, tener acceso y usar los sistemas financieros, así como el conocimiento técnico derivado de una educación financiera integral y permanente a lo largo de nuestras vidas nos permitirá tomar mejores decisiones y estar preparados ante eventualidades e imprevistos ya sea surgidos por ciclos económicos o circunstancias adversas personales. Es decir, el conocimiento no impedirá altibajos en el nivel de renta, errores por mal uso de instrumentos o productos financieros, sufrir algún delito que afecte nuestras finanzas o bien, vernos en aprietos financieros ocasionales. Lo que sí permitirá es afrontarlos atendiendo a mejores capacidades, competencias y preparación; evaluando a partir de escenarios posibles y haciendo uso de los mecanismos de protección porque educar a los usuarios del sistema financiero implica conocer sus derechos, herramientas e instituciones que los protegen y amparan ante actos indebidos, errores o abusos por parte de los prestadores de servicios al tiempo que éstos usuarios formados exigirán cada vez mayor transparencia, responsabilidad y calidad.

Adicional al conjunto de beneficios e impactos descritos en esta sección y enfatizando la relevancia de la educación financiera aducimos su efecto desencadenante puesto que mayores niveles de ahorro, inversión, gestión apropiada de deuda, diseño de planes financieros a corto y largo plazo apoyan indirectamente la economía porque generan oportunidades de inversión directa, emprendimientos productivos lo que finalmente se traduce en crecimiento, estabilidad y desarrollo económicos. Asimismo, se ha constatado que tiene efectos sobre el desarrollo y la pobreza, CEEY (2020) afirma que la educación financiera va más allá de los beneficios a las personas o a las empresas, es también un mecanismo de inclusión, de igualdad de oportunidades y de movilidad social.

Otros autores abordando la educación o la inclusión financiera encuentran evidencia de todo lo anterior, Allen et al. (2012; 49) afirman que el manejo de cuentas bancarias mejora el acceso a otros servicios e intermediarios financieros por lo que resulta altamente efectivo las políticas financieras encaminadas a que se oferten cuentas básicas a menores costos y requisitos para su obtención, dado que ello incide de manera fundamental en la inclusión financiera de los pobres. Cihák, Mare y Melecky (2016; 17) evidencian que la educación financiera puede generar una relación positiva entre la inclusión y la estabilidad al mejorar la inclusión financiera responsable que ayude al sistema financiero a 
aprovechar los beneficios de la escala económica y la diversificación del riesgo. Igualmente, al analizar la relación entre inclusión financiera y desarrollo Cull, Ehrbeck y Holle $(2014 ; 7)$ manifiestan que los servicios financieros son un medio para alcanzar un fin, con base en la revisión de pruebas que utilizan metodologías de investigación rigurosas parecen confirmar en general la convicción de los responsables políticos de que los mercados financieros inclusivos y eficientes, reducen los costes de las transacciones, estimulan la economía y mejoran el suministro de otras prestaciones sociales y soluciones innovadoras del sector privado.

\section{CONCLUSIONES}

La educación es un proceso paulatino con multiplicidad de beneficios de los cuales, dos de ellos resultan los más prometedores, la movilidad social y el progreso económico porque representan la posibilidad de cambio de las condiciones económicas y sociales, contrario a la perpetuidad de atroces condiciones de pobreza, desigualdad, discriminación. Dentro de este orden de ideas una propuesta que se ha puesto en marcha a nivel internacional es la promoción de la educación y la inclusión financieras atendiendo al argumento de que los conocimientos financieros son necesarios porque a diario se toman decisiones en la materia que impactan el patrimonio, repercutiendo en el largo plazo.

De este modo pretendemos realizar aportes para mejorar la comprensión del proceso formal de educar financieramente y analizar sus impactos a nivel transversal más allá del nivel académico logrado, a lo que se dedican las personas, sus nacionalidades, edades, géneros $u$ otras características. En este tenor el aporte se ha presentado desde dos perspectivas, la conceptual y la analítica. Proveyendo definiciones claras y específicos para educación, cultura e inclusión financiera. Igualmente explorando los efectos de la educación financiera desde el uso del dinero hasta la toma de decisiones que considera el entorno financiero, la estructura de los sistemas de entidades financieras y los mecanismos de protección y atención ante vulnerabilidades.

\section{REFERENCIAS}

Allen, F., A. Demirgüç-Kunt, L. Klapper y Martínez, M. (2012) The Foundations of Financial Inclusion: Understanding Ownership and Use of Formal Accounts. Documento de Trabajo, Policy Research No. 6290, Banco Mundial, EUA. Disponible en línea: https://openknowledge.worldbank.org/handle/10986/12203 [Consultado el: 28 de enero de 2021]

Armendáriz de Aghion, Beatriz y Morduch, Jonathan (2006) "Microfinance: where do we stand?", en Charles A. E. Goodhart, Financial Development and Economic Growth. Explaining the links, Inglaterra, Palgrave Macmillan.

ASIC. Australian Securities and Investments Commission (2018) Annual Highlights Report 2016-17. National Financial Literacy Strategy. Reporte Núm. 563, febrero. Australia. Disponible en línea: https://download.asic.gov.au/media/4661382/asic-national-financial-literacy-strategy-annualhighlights-report-2016-17.pdf [Consultado el: 28 de enero de 2021]

Banxico (2020) Introducción a los sistemas de pagos. Página electrónica del Banco Central en México. Disponible en línea: https://www.banxico.org.mx/sistemas-de-pago/introduccionsistemas-pago-tr.html [Consultado el: 28 de enero de 2021] 
Bosch, Mariano; Cofré, Fabián; González, Stephanie et.al. (2018) Educación financiera y previsional a través del Taller Ahorro desde Ahora: evidencia de una evaluación de impacto en Chile. Documento de Trabajo del Banco Interamericano de Desarrollo No. 1017, octubre.

Cihák, M., Mare, D. y Melecky, M. (2016) The Nexus of Financial Inclusion and Financial Stability: A Study of Trade-offs and Synergies. Documento de Trabajo, Policy Research No. 7722, Banco Mundial, EUA. Disponible en línea: http://documents. worldbank.org/curated/en/138991467994676130/The-Nexus-of-financialinclusion-and-financial-stability-a-study-of-trade-offs-and-synergies [Consultado el: 28 de enero de 2021]

CPEUM. Constitución Política de los Estados Unidos Mexicanos (1917) Congreso de la Unión, última modificación 24 de febrero de 2020. Diario Oficial de la Federación. Disponible en línea: http://www.diputados.gob.mx/LeyesBiblio/pdf/1 241220.pdf [Consultado el: 28 de enero de 2021]

CEE Comisión Económica Europea (2007) La Educación Financiera. Comunicado COM 808, 18 de diciembre.

CEEY (2020) Economía en México: la importancia de la educación financiera (I). México. Recuperado de: https://ceey.org.mx/que-tan-bien-funciona-la-economia-en-mexico/ [Consultado el: 28 de enero de 2021]

CONDUSEF Comisión Nacional para la Protección y Defensa de los Usuarios de Servicios Financieros (2009). ABC De Educación Financiera. Material de Difusión. México.

Cruz, Evangelina (2018) "Educación Financiera en los niños: una evidencia empírica". Revista Electrónica de Educación Sinéctica. Universidad Jesuita de Guadalajara, No. 52, Jul.-dic.

Cull, R., Ehrbeck, T y Holle, N. (2014) Financial Inclusion and Development: Recent Impact Evidence. Nota de Enfoque No. 92, Banco Mundial. EUA. Disponible en línea: http://documents. worldbank.org/curated/en/269601468153288448/Financial-inclusion-anddevelopment-recent-impact-evidence [Consultado el: 28 de enero de 2021]

FC. Financial Capability (2003) Anz Survey of Adult Financial Literacy in Australia Final Report. Documento de Trabajo. ANZ Banking Group y Roy Morgan Research, mayo, Australia. Disponible en línea: https://www.financialcapability.gov.au/files/anz-survey-of-adult-financialliteracy-2003.pdf [Consultado el: 28 de enero de 2021]

Habschick, Marco; Seidl, Britta y Evers, Jan (2007) "Survey of financial literacy schemes in the EU27". Reporte Final No. Markt/2006/26H, Hamburgo, noviembre. Disponible en línea: http://edufinanciera.com/wp-content/uploads/2014/02/report survey en.pdf [Consultado el: 28 de enero de 2021]

Hernández, Ariadna y Pérez, Soraya Coord. (2019) Visiones de la Educación Financiera: Análisis y Perspectivas. Cámara de Diputados LXIV Legislatura. México.

Jeftanovic P. (2001) "El dinero electrónico y la política monetaria". Revista Pharos, vol. 8, núm. 2, noviembre-diciembre. Universidad de Las Américas Santiago, Chile, pág. 89-98.

Jumpstart (2020) Historia de la Coalición Jumpstart. Página electrónica: https://www.jumpstart.org/who-we-are/about/history/ [Consultado el: 28 de enero de 2021]

Narro, José; Martuscelli, Jaime y Barzana, Eduardo (Coord.).(2012) Plan de diez años para desarrollar el Sistema Educativo Nacional. [En línea]. México: Dirección General de Publicaciones y Fomento Editorial, UNAM Disponible en línea: http://www.planeducativonacional.unam.mx [Consultado el: 28 de enero de 2021] 
OCDE. Organización para la Cooperación y el Desarrollo Económico. (2012) High-level Principles on National Strategies for Financial Education. Agosto. Francia. Disponible en línea: http://www.oecd.org/daf/fin/financial-education/OECD-INFE-Principles-National-StrategiesFinancial-Education.pdf [Consultado el: 28 de enero de 2021]

OCDE. Organización para la Cooperación y el Desarrollo Económico. (2005) Principles and Good Practices For Financial Education and Awareness. Directorate for Financial and Enterprise Affairs, Francia, Julio. Disponible en línea: https://www.oecd.org/daf/fin/financialeducation/35108560.pdf [Consultado el: 28 de enero de 2021]

Ortega, Manuel; Pino, Cristóbal; Merino, Melania y Ledrado, María (2009) Educación Financiera en Enseñanza Secundaria Obligatoria Nivel I Guía para el profesorado. Publicación en Convenio de Banco de España, Ministerio de Educación, Comisión Nacional del Mercado de Valores. España. Disponible en línea: https://www.oecd.org/finance/financial-education/50236319.pdf [Consultado el: 28 de enero de 2021]

Plan Nacional de Desarrollo 2010-2014- DNP. Colombia.

Robinson, Marguerite (2001) The Micro Finance Revolution: Sustainable Finance for the Poor. Open Society Institute, Banco Mundial. EUA.

SERNAC (S/A) Guía Familiar de Educación Financiera. Cuaderno de Apoyo del Programa Escolar de Educación Financiera. Ministerio de Economía, Fomento y Turismo. Chile

SEP (a) Secretaría de Educación Pública (2017) Modelo Educativo para la Educación Obligatoria. Diario Oficial de la Federación, 20 de junio. Disponible en: https://www.sep.gob.mx/work/models/sep1/Resource/15105/1/images/modelo educativo educ acion obligatoria.pdf [Consultado el: 28 de enero de 2021]

SEP (b) Secretaría de Educación Pública (2017) Modelo Educativo para la Educación Obligatoria. Educar para la libertad y la creatividad. Materiales educativos de la SEP, marzo Disponible en línea: https://www.gob.mx/cms/uploads/attachment/file/207252/Modelo Educativo OK.pdf [Consultado el: 28 de enero de 2021]

Sorenson, Kevin (2020) Discurso de presentación del Ministro de Finanzas de Canadá https://www.canada.ca/en/financial-consumer-agency/programs/financial-literacy/financialliteracy-strategy.html [Consultado el: 28 de enero de 2021]

Valenzuela, Mónica (2010) La Importancia de la Educación en la Actualidad. Guía con las bases metodológicas e investigadoras para una mejora de la educación. Documento de Trabajo Eduinnova. España.

WWB. World Women Bank (2008) Manual de Educación Financiera. Ministerio de Educación, Política Social y Deporte, España 